\title{
Iterative meshfree methods for the Helmholtz equation
}

\author{
O. von Estorff \& C. Wenterodt \\ Institute of Modelling and Computation, \\ Hamburg University of Technology, Germany
}

\begin{abstract}
For acoustic computations in the mid-frequency range the finite element method (FEM) is a well-known standard tool. Unfortunately, for increasing frequencies, i.e. higher wavenumbers, the FEM suffers from the so-called pollution effect which is mainly a consequence of the dispersion, meaning that the numerical wavenumber and the exact wavenumber disagree. Using meshfree methods as, e.g., the radial point interpolation method (RPIM) or the element-free Galerkin method (EFGM) can reduce this effect significantly.

Moreover, meshfree methods allow the usage of shape functions that can be adapted to the differential equation to be solved. Consequently, an iterative method can be derived, which uses a standard meshfree method to compute a first approximation for the given problem. In a second step this approximation is taken to construct new shape functions that are able to better reproduce the wave-like character of the solution. If a few requirements are considered, this method leads to better results in a more efficient way.

In this paper two meshfree methods, namely an iterative RPIM and an iterative EFGM, are examined. The methods are compared to the FEM and restrictions for an efficient applicability are shown.

Keywords: Helmholtz equation, meshfree methods, acoustics, iterative method, EFGM, RPIM.
\end{abstract}

\section{Introduction}

In recent years meshfree methods have attracted more and more attention in several disciplines of computational mechanics [1]. Especially in the area of computational acoustics, it is well-known that the standard methods as, e.g., the finite 
element method (FEM) or the boundary element method (BEM) possess some drawbacks. The FEM suffers from the pollution effect. Especially for high wavenumbers the results become inaccurate since the dispersion error increases [2]. For the BEM an increasing number of elements leads to a significantly longer computation time, since the system matrices are fully populated and possibly nonsymmetric [3].

In contrast, meshfree methods offer some advantages. For the construction of the shape functions no mesh is needed. Instead, shape functions are constructed with respect to field points which can be arbitrarily distributed inside the domain under consideration. Amongst others, this makes the implementation of adaptive methods rather straightforward.

With respect to acoustic computations, in comparison to the FEM the dispersion effect can be drastically reduced for the element free Galerkin method (EFGM) [4] as well as for the radial point interpolation method (RPIM) [5]. Furthermore, the shape functions of the meshfree methods can be easily adapted to the problem to be solved. Consequently, Lacroix et al. [6] introduced an iterative EFGM which is based on problem-dependent shape functions. In a first step, an approximation for the sound pressure distribution inside the given domain is computed using a standard EFGM. From this solution the phase distribution can be obtained and then be used for the construction of new phase-dependent shape functions. These shape functions are better suited to reproduce the wave-like character of the solution and thus the results can be improved.

In this paper the method of Lacroix et al. has been further examined. In fact, own investigations have shown, that the accuracy of the iterative EFGM is highly dependent on the numerical integration scheme. Furthermore, different weight functions have been introduced and compared in order to optimize the method. Additionally, an iterative RPIM has been implemented; it will be compared to the iterative EFGM.

\section{Acoustics}

The propagation of pressure waves in an acoustic fluid inside a domain $\Omega$ is governed by the wave equation which can be derived using the balance of mass and momentum as well as the ideal gas law, provided that the state variables pressure $P$, density $\rho$, and velocity $v$ experience only small variations. For time harmonic waves of frequency $\omega$ the sound pressure $p$ is the solution of the Helmholtz equation

$$
\nabla^{2} p+k^{2} p=0
$$

with wavenumber $k=\omega / c$ and speed of sound $c$. The boundary $\Gamma$ of the fluid domain $\Omega$ is decomposed into three distinct regions $\Gamma_{D}, \Gamma_{N}$, and $\Gamma_{R}$. The associated boundary conditions are

- Dirichlet boundary conditions on $\Gamma_{D}$ :

$$
p=\bar{p}
$$


- Neumann boundary conditions on $\Gamma_{N}$ :

$$
\frac{\partial p}{\partial \mathbf{n}}=-i \omega \rho \bar{v}_{n}
$$

- Robin boundary conditions on $\Gamma_{R}$ :

$$
\frac{\partial p}{\partial \mathbf{n}}=-i \omega \rho A_{n} p
$$

Here, $A_{n}$ represents the admittance, $\mathbf{n}$ is the normal vector and $v_{n}$ the normal velocity.

\section{Meshfree methods}

In comparison to mesh-based methods as, e.g., the FEM, meshfree methods do not need a mesh for the representation of the domain $\Omega$ to be examined. In $\Omega$ and on its boundary $\Gamma$, field points $\mathbf{x}_{i}$ can be arbitrarily distributed. An approximation of the sound pressure $p^{h}$ at an arbitrary point $\mathbf{x}_{Q}$ in $\Omega$ is obtained by interpolation of the form

$$
p^{h}\left(\mathbf{x}_{Q}\right)=\sum_{i=1}^{n} \Phi_{i}\left(\mathbf{x}_{Q}\right) p_{i} .
$$

Here, $p_{i}$ is the coefficient of the solution vector $\mathbf{p}$ belonging to field point $\mathbf{x}_{i}$ and $\Phi_{i}$ is the associated shape function which is constructed using all $n$ field points that influence $\mathrm{x}_{Q}$. In order to obtain an equation system that can be solved for the coefficient vector $\mathbf{p}$, the shape functions are substituted into the weak form of the Helmholtz equation both as test and as trial functions. The resulting equations have to be integrated numerically. For this reason a background mesh has to be introduced, which can be chosen independently of the field point distribution and which does not have to represent the domain $\Omega$.

\section{Shape functions}

\subsection{EFGM shape functions}

In order to obtain the shape functions of the EFGM, the unknown sound pressure $p^{h}$ is interpolated by

$$
p^{h}(\mathbf{x})=\sum_{i=1}^{n} P_{i}(\mathbf{x}) a_{i}(\mathbf{x})=\mathbf{P}^{T}(\mathbf{x}) \mathbf{a}(\mathbf{x}) .
$$

Here, $\mathbf{P}^{T}(\mathbf{x})$ denotes a basis, which can be chosen as e.g.

$$
\mathbf{P}^{T}(\mathbf{x})=\left[\begin{array}{lll}
1 & x & y
\end{array}\right]
$$

in the two-dimensional case. The coefficient vector $\mathbf{a}(\mathbf{x})$ is determined by minimization of a functional such that the approximation $p^{h}\left(\mathbf{x}_{i}\right)$ at the field points 
yields the best possible agreement with the according coefficients $p_{i}$ of the solution vector. This leads to

$$
\mathbf{a}(\mathbf{x})=\mathbf{A}^{-1}(\mathbf{x}) \mathbf{B}(\mathbf{x}) \mathbf{p}
$$

with

$$
\begin{aligned}
\mathbf{A}(\mathbf{x}) & =\sum_{i=1}^{n} w_{i}(\mathbf{x}) \mathbf{P}\left(\mathbf{x}_{i}\right) \mathbf{P}^{T}\left(\mathbf{x}_{i}\right) \\
\mathbf{B}(\mathbf{x}) & =\left[w_{1}(\mathbf{x}) \mathbf{P}\left(\mathbf{x}_{i}\right), \ldots, w_{n}(\mathbf{x}) \mathbf{P}\left(\mathbf{x}_{n}\right)\right] .
\end{aligned}
$$

In eqns (9) and (10) the weight functions $w_{i}(\mathbf{x})$ can be chosen, e.g., as the cubic spline

$$
w_{i}(r)= \begin{cases}\frac{2}{3}-4 r^{2}+4 r^{3} & \text { for } r \leq \frac{1}{2} \\ \frac{4}{3}-4 r+4 r^{2}-\frac{4}{3} r^{3} & \text { for } \frac{1}{2}<r \leq 1 \\ 0 & \text { for } r>1\end{cases}
$$

the quartic spline

$$
w_{i}(r)= \begin{cases}1-6 r^{2}+8 r^{3}-3 r^{4} & \text { for } r \leq 1 \\ 0 & \text { for } r>1\end{cases}
$$

or the exponential function

$$
w_{i}(r)= \begin{cases}\frac{\exp \left(-(2 r)^{2}\right)-\exp (-4)}{1-\exp (-4)} & \text { for } r \leq 1 \\ 0 & \text { for } r>1\end{cases}
$$

with $r$ denoting the distance between point $\mathbf{x}$ and field point $\mathbf{x}_{i}$ scaled by the influence radius $r_{i n f l}$, i.e.

$$
r=\frac{\left\|\mathbf{x}-\mathbf{x}_{i}\right\|}{r_{i n f l}}
$$

Substituting eqn (8) into eqn (6) yields

$$
p^{h}(\mathbf{x})=\mathbf{P}^{T}(\mathbf{x}) \mathbf{A}^{-1}(\mathbf{x}) \mathbf{B}(\mathbf{x}) \mathbf{p}=\mathbf{\Phi}(\mathbf{x}) \cdot \mathbf{p}
$$

with the vector of shape functions

$$
\mathbf{\Phi}(\mathbf{x})=\mathbf{P}^{T}(\mathbf{x}) \mathbf{A}^{-1}(\mathbf{x}) \mathbf{B}(\mathbf{x}) .
$$




\subsection{RPIM shape functions}

The shape functions for the RPIM can be obtained by an ansatz of the form

$$
\begin{aligned}
p^{h}\left(\mathbf{x}, \mathbf{x}_{Q}\right) & =\sum_{i=1}^{n} R_{i}(\mathbf{x}) a_{i}\left(\mathbf{x}_{Q}\right)+\sum_{j=1}^{n} P_{j}(\mathbf{x}) b_{j}\left(\mathbf{x}_{Q}\right) \\
& =\mathbf{R}^{T}(\mathbf{x}) \mathbf{a}\left(\mathbf{x}_{Q}\right)+\mathbf{P}^{T}(\mathbf{x}) \mathbf{b}\left(\mathbf{x}_{Q}\right) .
\end{aligned}
$$

Here, $R_{i}$ is a radial basis function which can be chosen as, e.g., the multiquadrics

$$
R_{i}(r)=\left(r^{2}+\left(\alpha_{c} d_{c}\right)^{2}\right)^{q}
$$

where $r$ denotes the distance between point $\mathbf{x}$ and field point $\mathbf{x}_{i}$. The characteristic length $d_{c}$ is the average field point spacing in the influence domain and $\alpha_{c}$ and $d_{c}$ can be adapted to the problem. The additional polynomial basis $\mathbf{P}^{T}(\mathbf{x})$ in eqn (17) ensures the consistency of the method and can be chosen as for the EFGM in eqn (7). The unknown coefficients $a_{i}$ and $b_{j}$ are chosen such that eqn (17) is fulfilled at all $n$ field points that influence point $\mathbf{x}_{Q}$.

It should be noted that the RPIM shape functions possess the Kronecker delta property, which is not the case for the EFGM shape functions. The coefficient vector $\mathbf{p}$ contains sound pressure values at the field points only if the method fulfills the Kronecker delta property. More information about the construction of meshfree shape functions and their properties can be found in [1].

\section{Iterative meshfree method}

Meshfree shape functions of the RPIM and the EFGM can be easily adapted to the problem to be solved. This concept has, e.g., been used for the examination of crack tip fields [7]. For acoustic problems that are described by the Helmholtz equation, the sound pressure at a point $\mathrm{x}$ can be written as

$$
p(\mathbf{x})=\hat{P}(\mathbf{x})(\cos \theta(\mathbf{x})+\mathrm{i} \sin \theta(\mathbf{x}))
$$

with $\hat{P}(\mathbf{x})$ being the amplitude and $\theta(\mathbf{x})$ being the phase of the wave. The polynomial basis in eqn (7) or (17) is now substituted by the basis

$$
\mathbf{P}^{T}(\mathbf{x})=\left[\begin{array}{lll}
1 & \cos \theta(x, y) & \sin \theta(x, y)
\end{array}\right] .
$$

Provided that the phase is exactly known in the whole domain $\Omega$, the shape functions constructed with the basis (20) can interpolate the sound pressure exactly. Since the phase is generally unknown, a first approximation of the sound pressure $p_{1}^{h}(\mathbf{x})$ is computed using a standard EFGM or RPIM, respectively. From this pressure field an approximation of the phase distribution can be obtained and then be used for the construction of the adapted shape functions. Using the adapted shape functions, a second approximation $p_{2}^{h}(\mathbf{x})$ is computed. This algorithm can either be repeated for a predefined number of iterations or aborted if a convergence criterion is reached. 
The method described above has been proposed and tested by Lacroix et al. [6] for the EFGM. Own investigations have shown that the success of the iterative method is highly dependent on the quality of the numerical integration. Furthermore the methodology can be optimized if proper weight functions are used. Moreover, the applicability of phase-dependent RPIM shape functions has not yet been investigated.

\section{Numerical investigations}

In the following, the iterative methods as well as the FEM are applied to an acoustic problem that can also be solved analytically. The square domain $\Omega$ shown in figure 1 with edge length $L=1 \mathrm{~m}$ is investigated. At $x=y=0$ a Dirichlet boundary condition with $p_{0}=1 \mathrm{~N} / \mathrm{m}^{2}$ is prescribed. On all four edges Robin boundary conditions are chosen such that the analytical solution is a plane wave of the form

$$
p(x, y)=\cos (k(x \cos \beta+y \sin \beta))+\mathrm{i} \sin (k(x \cos \beta+y \sin \beta)) .
$$

Here, $\beta$ denotes the propagation angle and is defined as $\beta=20^{\circ}$ in the following. The fluid properties are chosen as density $\rho=1.247 \mathrm{~kg} / \mathrm{m}^{3}$ and speed of sound $c=343 \mathrm{~m} / \mathrm{s}$ which correspond to air.

Figure 2 shows the $L_{2}$ error of the iterative methods for a wavenumber $k=$ $1 \frac{1}{\mathrm{~m}}$ and field point distance $h_{f p}=0.1 \mathrm{~m}$. The background mesh is chosen in accordance to the field point distribution, i.e. the size of the background cells is $h_{b g}=0.1 \mathrm{~m}$. The number of gauss points in each cell is $n_{q p}=10 \times 10$. Additionally, the error of the FEM is shown. For the FEM no iterative method has been implemented and therefore the error of the FEM is constant here.

It is obvious that the EFGM yields the best result. The first iteration which is carried out using the standard EFGM already leads to an error which is only $0.4 \%$

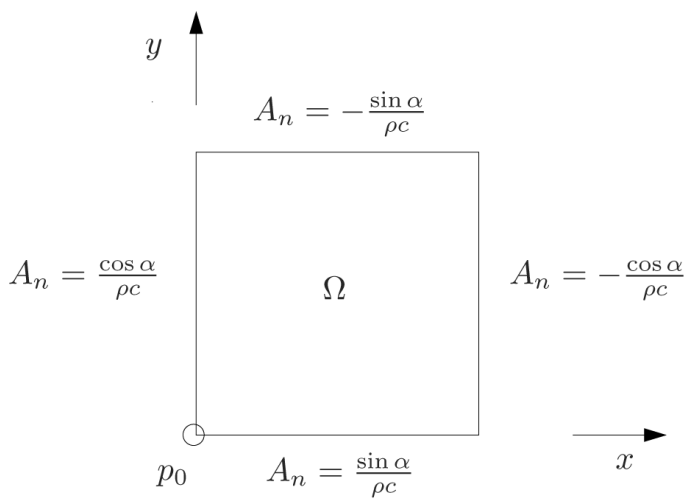

Figure 1: Acoustic domain $\Omega$ with Robin and Dirichlet boundary conditions. 


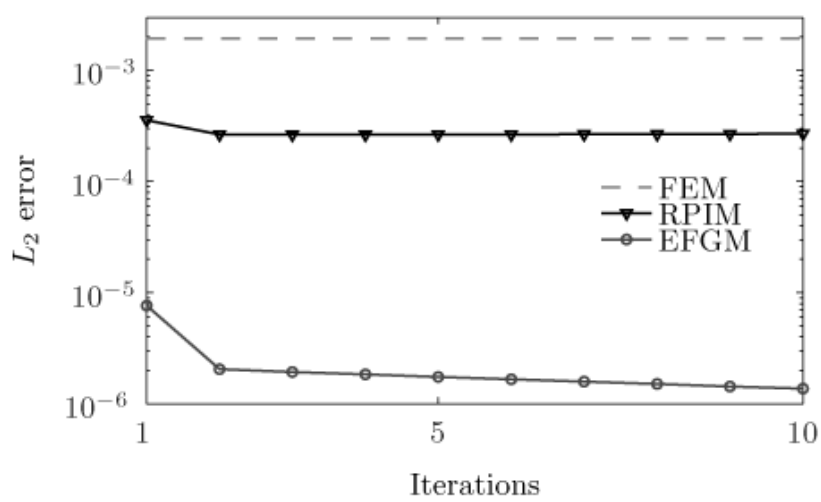

Figure 2: $L_{2}$ error of the RPIM, EFGM, and FEM for $k=1 \frac{1}{\mathrm{~m}}$ and $h_{f p}=0.1 \mathrm{~m}$.

of the FEM error. The RPIM leads to at least $18 \%$ of the FEM error. During the following iterations the error of the EFGM and the RPIM can be further reduced, although the effect is much clearer for the EFGM.

In order to show the local effect of the iterative EFGM figure 3(a) contains the error distribution on $\Omega$ for the first iteration which corresponds to the standard EFGM. Furthermore in figure 3(b) the error distribution after the second iteration is shown. The error could be significantly reduced. For comparison the error of an optimal EFGM is presented in figure 3(c). Here, "optimal EFGM" means, that the shape functions are constructed using the phase distribution of the analytical solution. As already mentioned before, the resulting shape functions are dispersion free and are able to represent the solution exactly. The error of the optimal EFGM almost vanishes but it is not zero everywhere. The reasons for this will be explained later.

Several computations have shown, that the capabilities of the iterative EFGM are highly dependent on the quality of the numerical integration. For the standard EFGM the numerical integration does not have a significant influence on the results, but the phase-dependent shape functions appear to be very sensitive to the choice of the background mesh and the numerical integration order. Figure 4 shows the $L_{2}$ error of the EFGM for different background meshes. The size of the background cells $h_{b g}$ as well as the number of integration points in each cell $n_{q p}$ have been varied. If a coarse background mesh with a low integration order (in this case $h_{b g}=0.1 \mathrm{~m}$ and $n_{q p}=4 \times 4$ ) is chosen, the iterative method even deteriorates the results of the standard EFGM in the following iterations.

Similar investigations for the RPIM have shown that the background mesh and integration order do not influence the quality of the method. On the other hand, the results of the RPIM cannot keep up with the EFGM. For this reason the following analysis is carried out for the EFGM only. 

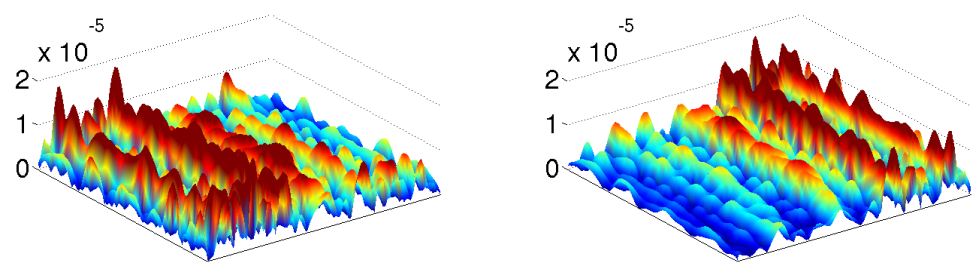

(a) Standard EFGM (first iteration)
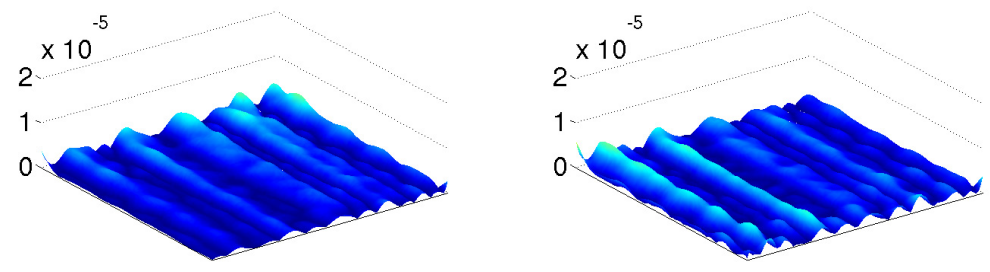

(b) Standard EFGM (second iteration)
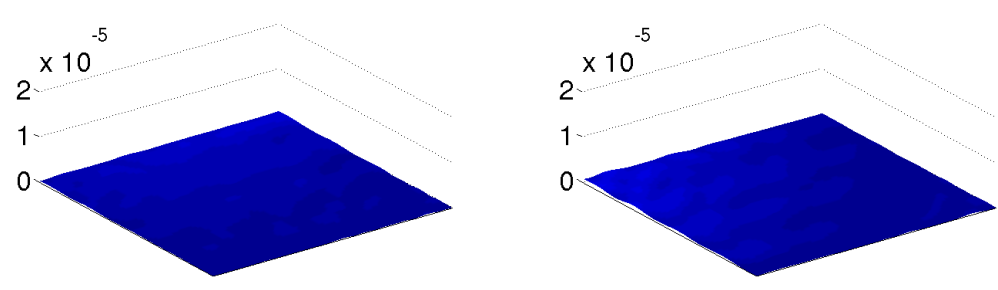

(c) Optimal EFGM

Figure 3: Error distribution of the standard, iterative, and optimal EFGM for $k=1 \frac{1}{\mathrm{~m}}, h_{f p}=0.1 \mathrm{~m}, h_{b g}=0.1 \mathrm{~m}$, and $n_{q p}=10 \times 10$ (left: real part, right: imaginary part of the error).

Up to now, only 10 iterations have been considered. Figure 5 shows the results of the iterative EFGM (EFGM iterative) for 10 as well as 100 iterations. For comparison, the $L_{2}$ error of the optimal EFGM (EFGM opt) is plotted. This means that for the first iteration the optimal EFGM with shape functions depending on the analytical phase distribution has been used and in the subsequent iterations the shape functions have been computed using the phase of the solution obtained from the last iteration.

The optimal EFGM reaches an $L_{2}$ error of $4 \cdot 10^{-7}$ in the first iteration. Due to numerical instabilities this value oscillates slightly during the next iterations. The iterative EFGM converges towards a limit of $7 \cdot 10^{-7}$.

In order to assess the influence of the numerical integration on the results in a more objective way, the optimal EFGM is further examined. In theory, the optimal EFGM should yield an optimal solution because the shape functions interpolate the sound pressure exactly. Nevertheless, the solution is not exact due to errors of the numerical integration and other instabilities that result from e.g. matrix inversions. Figure 6 shows the $L_{2}$ error of the optimal EFGM for different sizes $h_{b g}$ of the 


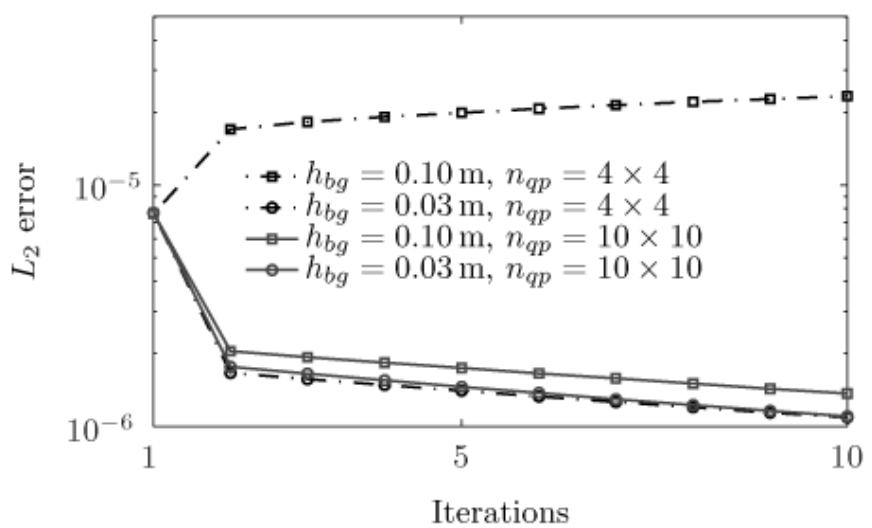

Figure 4: $L_{2}$ error for different background meshes for $k=1 \frac{1}{\mathrm{~m}}$ and $h_{f p}=0.1 \mathrm{~m}$.
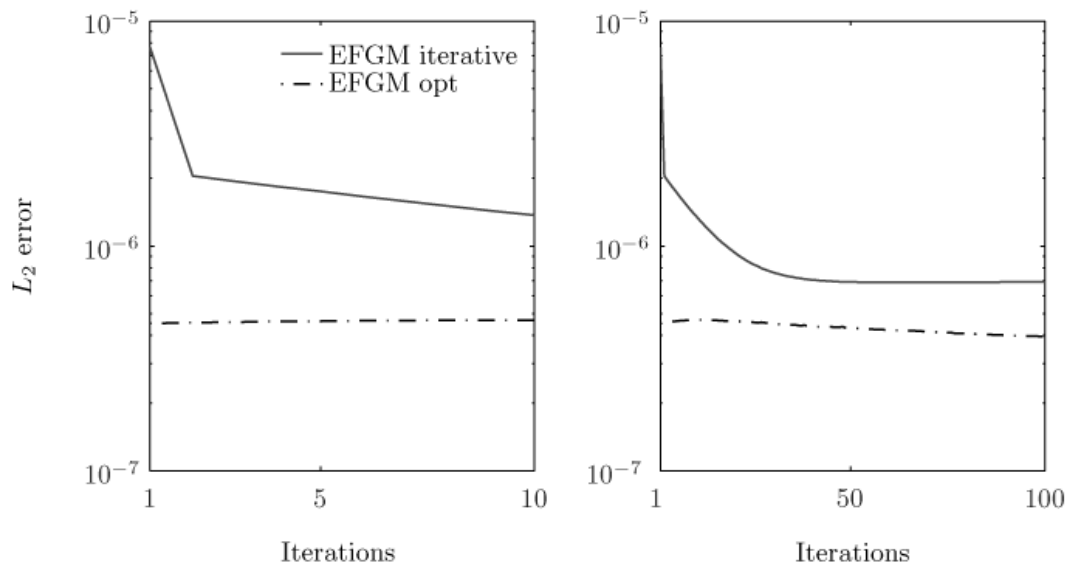

Figure 5: $L_{2}$ error of the EFGM and the optimal EFGM for $k=1 \frac{1}{\mathrm{~m}}, h_{f p}=h_{b g}=$ $0.1 \mathrm{~m}$, and $n_{q p}=10 \times 10$.

background cells (left) and different numbers of gauss points $n_{q p}$ (right). The influence of the integration scheme can be clearly seen: If the cells are chosen too coarse or if the integration order is too low, the optimal EFGM can only yield an $L_{2}$ error of about $10^{-4}$ to $10^{-3}$. In contrast, the $L_{2}$ error can be reduced to less than $10^{-8}$ if the integration is improved. Furthermore, the method leads to better results if the size of the background cells is a multiple of the field point spacing and vice versa.

Finally, the influence of different weight functions $w_{i}(\mathbf{x})$ on the results of the EFGM are examined. Up to now, all computations have been performed using the 
166 Boundary Elements and Other Mesh Reduction Methods XXXII
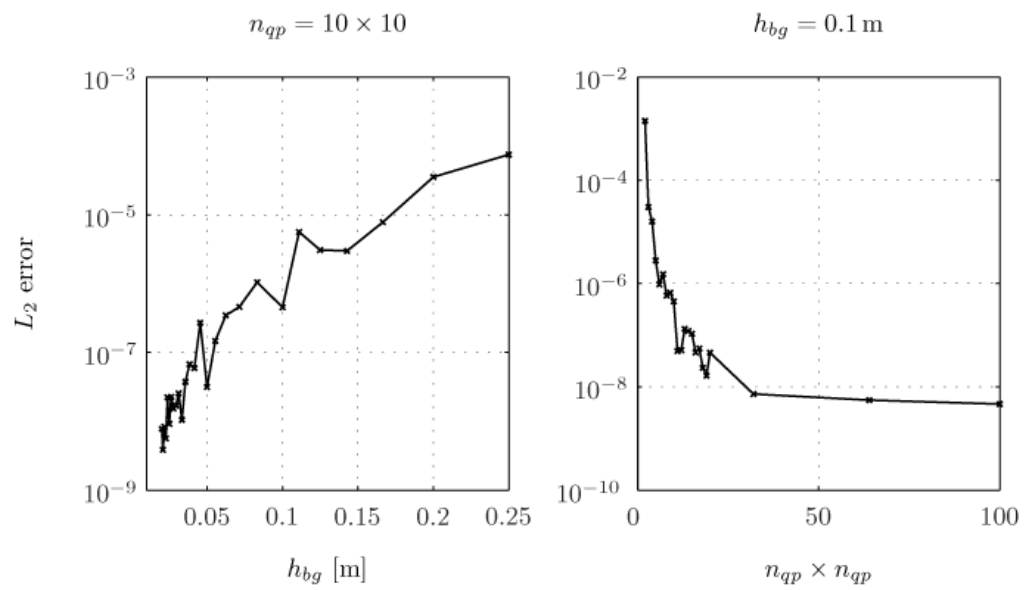

Figure 6: $L_{2}$ error of the optimal EFGM $\left(k=1 \frac{1}{\mathrm{~m}}, h_{f p}=0.1 \mathrm{~m}\right)$.
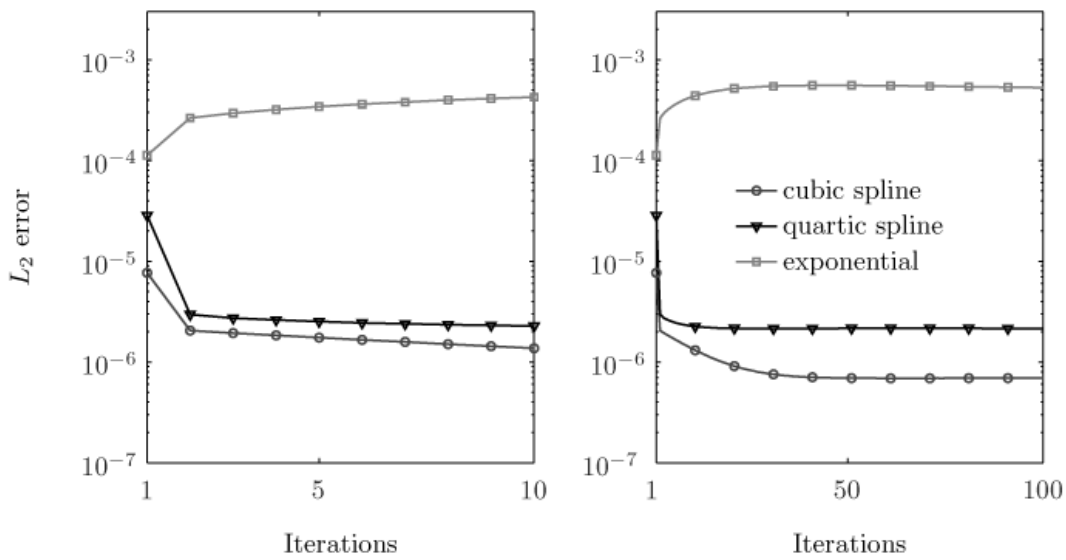

Figure 7: $L_{2}$ error of the EFGM for different weight functions for $k=1 \frac{1}{\mathrm{~m}}$, $h_{f p}=h_{b g}=0.1 \mathrm{~m}$, and $n_{q p}=10 \times 10$.

cubic spline. In [6] Lacroix et al. proposed exponential weight functions. Figure 7 shows the $L_{2}$ error of the iterative EFGM if the cubic spline in eqn (11), the quartic spline in eqn (12) and the exponential function in eqn (13) are used. Obviously the weight function has a considerable influence on the error of not only the standard EFGM (first iteration) but also of the iterative EFGM. In the considered case the cubic spline weight functions yield the lowest error. In contrast, the iterative EFGM with exponential weight function even increases the error of the standard EFGM. 


\section{Conclusions}

In this contribution, two meshfree methods, namely the EFGM and the RPIM, have been applied to a two-dimensional acoustic problem. An iterative method based on shape functions that are adapted to the problem has been implemented and investigated especially with respect to the numerical integration scheme. Furthermore, for the EFGM, the influence of different weight functions has been examined.

The EFGM as well as the RPIM lead to a lower $L_{2}$ error than the FEM for similar discretizations. The iterative methods further reduce this error, but only if the numerical integration scheme is chosen appropriately. The influence of the background mesh and integration order on the iterative method is less considerable for the RPIM. On the other hand, the EFGM leads to much lower errors than the RPIM.

Furthermore, the influence of different weight functions on the quality of the solution has been examined. For the considered example, the cubic spline performed best whereas the exponential weight function did not yield reasonable results.

In order to be able to use the iterative methods in practice, further investigations are necessary, e.g., application to real life problems and the analysis of the computation time.

\section{References}

[1] Liu, G., Mesh Free Methods: Moving beyond the Finite Element Method. CRC Press: Boca Raton, 2003.

[2] Deraemaeker, A., Babuška, I. \& Bouillard, P., Dispersion and pollution of the FEM solution for the Helmholtz equation in one, two and three dimensions. International Journal for Numerical Methods in Engineering, 46(4), pp. 471499, 1999.

[3] von Estorff, O., (ed.) Boundary Elements in Acoustics: Advances and Applications. WIT Press: Southampton, 2000.

[4] Suleau, S., Deraemaeker, A. \& Bouillard, P., Dispersion and pollution of meshless solutions for the Helmholtz equation. Computer Methods in Applied Mechanics and Engineering, 190(5), pp. 639-657, 2000.

[5] Wenterodt, C. \& von Estorff, O., Dispersion analysis of the meshfree radial point interpolation method for the Helmholtz equation. International Journal for Numerical Methods in Engineering, 77(12), pp. 1670-1689, 2009.

[6] Lacroix, V., Bouillard, P. \& Villon, P., An iterative defect-correction type meshless method for acoustics. International Journal for Numerical Methods in Engineering, 57(15), pp. 2131-2146, 2003.

[7] Fleming, M., Chu, Y., Moran, B. \& Belytschko, T., Enriched element-free Galerkin methods for crack tip fields. International Journal for Numerical Methods in Engineering, 40, pp. 1483-1504, 1997. 